\title{
Evaluation of Antiplatelet Activity of Phenolic Compounds by Flow Cytometry
}

\author{
Konstantinos D. Kyriakidis, Eyrysthenis G. Vartholomatos, and Georgios S. Markopoulos
}

\section{ABSTRACT}

Platelets play a pivotal role in coagulation, or clot formation, resulting in haemostasis, after endothelium injury. Disturbance of platelet activation may lead to pathologic thrombosis. Platelet activation and aggregation are common factors in atherothrombotic events, critical in the atherothrombotic process, and cardiovascular diseases. Several drugs are being used for antiplatelet therapy to prevent and/or treat atherosclerosis and cardiovascular diseases. Synthetic antiplatelet drugs hold possible undesired health consequences (cardiovascular diseases, carcinogenicity, etc.), advocating their replacement with natural, effective, and non-toxic compounds. Many phenolic compounds are created as secondary metabolites of plants, are found in many fruits and vegetables, and constitute a wide family of high-added-value molecules. Their biological activities include antioxidant, anti-platelet, and anti-inflammatory action. Based on the above, we examined five phenolic compounds (ellagic acid, ferulic acid, gallic acid, quercetin, and kaempferol) for their effect on platelet reactivity in whole blood samples using flow cytometry.

Quantification of activated platelet marker CD62-P by flow cytometry showed that all five compounds inhibited platelet activation in vitro, induced by adenosine diphosphate (ADP) and collagen. Interestingly, based on the $\mathrm{IC}_{50}$ values obtained for expression of CD62-P, among ellagic, ferulic, and gallic acid, gallic acid showed significantly higher inhibition than the other two. Kaempferol found to be a more potent inhibitor than quercetin, following previously reported results from aggregometry. Results obtained from our flow cytometry screening indicate antiplatelet activity from novel phenolic compounds and their potential use as drugs for thrombosis and cardiovascular diseases.

Keywords: phenolic acids, flavonols, platelet biomarkers, P-selectin, flow cytometry.

\section{INTRODUCTION}

Haemostasis is an important physiological process, wherein activated platelets adhere to the injured vessel wall, form aggregates to stop bleeding and create a barrier for infections. The primary step in haemostasis is the adhesion of platelets to the extracellular matrix and is mediated by the interaction between the glycoprotein (GPIbeta/V/IX) receptor complex on the platelet surface to von Willebrand factor and GPVI and GPIalpha to collagen at sites of vascular injury [1]. The above interaction is required for the initial adhesion of platelets to sub-endothelium. Platelet activation is stimulated by bound platelets secretion products and local prothrombotic factors, leading to haemostasis. Collagen, adenosine diphosphate (ADP), epinephrine, serotonin, thromboxane $\mathrm{A}_{2}$ and thrombin facilitate platelet activation [1]. More specifically, they recruit platelets from circulation, platelets change shape and release several pro-inflammatory molecules as P-selectin and CD40 ligand, convert GPIb/IIIa complex (a central platelet receptor mediating platelet aggregation) into its
Submitted : February 2, 2021

Published : February 18, 2021

ISSN: 2593-8339

DOI: $10.24018 /$ ejmed.2021.3.1.703

Konstantinos D. Kyriakidis *

Colchester Hospital University Foundation Trust, UK.

(e-mail: kkonstantis@yahoo.com)

Eyrysthenis G. Vartholomatos

Laboratory of Molecular Biology,

University Hospital of Ioannina, Greece.

Georgios S. Markopoulos

Laboratory of Molecular Biology,

University Hospital of Ioannina, Greece.

*Corresponding Author

active form, which allows platelet aggregation. In arterial thrombosis, increased platelet activation can be measured indirectly, through quantification of activation markers on their cell surface. These platelet biomarkers are of great importance, as they may predict thrombotic situations. Among these, P-selectin CD62P, CD63, CD40L GPIIb/IIIA are well studied and extensively used. CD62P is found in the $\alpha$-granules of platelets. Presence of CD62-P on the surface of the platelets indicates an activated platelet state while their absence implies a resting state [2]. Granulophysin, CD63, moves from granules and lysosomes to the plasma membrane after platelet activation. CD40L also migrates to the platelet surface from a-granules a similar manner to Pselectin. Platelet reactivity can be easily evaluated, recording CD62-P levels on platelets stimulated by using classic agonists such as collagen and ADP. Estimation of surface-bound CD62P alone or/in combination with CD63 or CD40L, by flow cytometric analysis is also widely used for diagnosis of platelet activation state in ex vivo patientderived samples. Elevated levels of CD62-P were detected in several diseases such as peripheral artery and ischemic 
stroke [3], [4].

Among the most potent antiplatelet drugs are either cyclooxygenase-1(COX-1) inhibitors such as aspirin, ADP agonists such as ticlopidine and clopidogrel [5]-[7] or/and membrane glycoprotein antagonists such as tirofiban [8]. Besides a strong action in certain cases, several undesired side effects on the gastrointestinal system have been recorded. Thus, the need for new antiplatelet drugs is very important. Natural products may be a good alternative since the impact of dietary polyphenols on aggregation has been widely studied [9]. Natural phenolic acids (gallic, ellagic, ferulic) and flavonols (quercetin and kaempferol), isolated from wine wastes, sea buckthorne berries, and pomegranate wastes and also present in many fruits and vegetables, are reported to exert significant radical scavenging activity, antiplatelet and anti-inflammatory activity, in vitro [10], [11]. Here, the effect of these five phenolic compounds was also examined on platelet reactivity by flow cytometric analysis in an attempt to gain further insight on their antiplatelet impact.

\section{MATERIAL AND MethodS}

\section{A. Chemical Compounds}

Phenolic acids (gallic, ellagic, ferulic) and quercetin were purchased from Aldrich-Sigma Chem. Co (Germany) and kaempferol from Fluka Biochimica (Germany). Collagen and ADP were purchased from Aldrich-Sigma Chem. Co (Germany) and added at a concentration of $3.2 \mathrm{mg} / \mathrm{ml}$ and $6.5 \mathrm{mM}$ respectively, were used for platelet activation in our flow cytometry experiments.

\section{B. Flow Cytometry}

Flow cytometric measurements were performed in a FACScalibur cytometer (Becton Dickinson), based on the following protocol. In $40 \mu \mathrm{l}$ of PBS buffer, $10 \mu \mathrm{l}$ hirudintreated blood was added followed by the addition of the phenolic compound at four different concentrations ranging from 0.01 to $2.0 \mathrm{mM}$. After incubation for $10 \mathrm{~min}$ at $37{ }^{\circ} \mathrm{C}$, the respective agonist $(20 \mu \mathrm{l})$ was added and incubated for another $5 \mathrm{~min}$ at room temperature. Then, the antibody mixture (CD) was added, followed by a final incubation of $10 \mathrm{~min}$ at room temperature in the dark. In the final step $300 \mu \mathrm{l}$ red-blood cell lysis buffer (VersaLyse, Beckman coulter) was added, the mixture stayed for $10 \mathrm{~min}$ at room temperature before analysis by flow cytometry. In all cases 20.000 platelets (events) in the electronic gate of platelets. To minimize in vitro artifacts, fixation or washing procedures were omitted. The absorbance of each sample was carried out with a low flow $(12 \pm 3 \mathrm{ml} / \mathrm{min})$, while the data were processed with the Cell Quest software. Activated platelets were analyzed by quantifying the expression of the activated-platelet marker CD62-P PE (Becton Dickinson), having as reference CD61PerCP (Becton Dickinson) and CD41a FITC (Becton Dickinson) platelet markers. For calculations of activated platelets, we used the mean expression of activated CD62-P, at different concentrations of the substances. The $\mathrm{IC}_{50}$ values were determined and the reported ones are the mean values of three different experiments.

\section{RESUlTS AND DisCUSSION}

Recombinant hirudin was used as anticoagulant in the series of experiments reported here. Sodium citrate, heparin, and hirudin are the most studied ones. Of the anticoagulants investigated so far, the selective thrombin inhibitor hirudin is considered as the most suitable anticoagulant for studies of platelet aggregation in vitro in whole blood [12], [13]. As it has been reported ADP- and collagen-induced aggregation was significantly lower in citrated blood compared to hirudin-treated blood, reflecting the importance of extracellular calcium for platelet function [12]. Recently, Kalb et al [14] compared platelet aggregability by Multiple Electrode Aggregation method, in whole blood stored in citrate, heparin, and direct thrombin inhibitors, and found no significant differences between samples containing direct thrombin inhibitors and samples containing heparin at baseline. In contrast, aggregation, by this method, was significantly impaired in citrate-anticoagulated blood.

Platelets were gated based on forward and side scatter (FSS/SSC) and positivity for platelet-specific CD61 antigen (integrin GPIIb). The presence of CD62-P on the surface of the platelets indicates an activated-platelets state while their absence implies a resting state of the platelets [15]. For calculations of activated platelets, we used the mean expression of activated platelets, for all examined compounds, using ADP or/and collagen as platelet agonists. ADP-induced P-selectin expression levels, at the various concentrations of each sample, were recorded and thereafter the percentage of inhibition at a certain concentration of compound was estimated. These values were used for the determination of $\mathrm{IC}_{50}$ values.<smiles>O=c1c(O)c(-c2ccc(O)cc2)oc2cc(O)cc(O)c12</smiles><smiles>O=C(O)c1cc(O)c(O)c(O)c1</smiles><smiles>COc1cc(/C=C/C(=O)O)ccc1O</smiles>

Fig. 1. Chemical structures of phenolic compounds examined: of kaempferol 1, quercetin 2, gallic acid $\mathbf{3}$ and ferulic acid 4.

The chemical structures of kaempherol (1), quercetin (2), gallic acid (3) and ferulic acid (4) are shown in Fig. 1. Ellagic acid, the fifth compound examined, as the dimer of gallic acid is not shown in the figure. All these are common secondary metabolites of plants and beverages as fruits, oils, nuts, vegetables, wine, coffee etc. [16]. Ferulic acid has been used to treat thrombosis [17], [18]. Here, ferulic acid found to inhibit platelet aggregation induced by both collagen and $\mathrm{ADP}$ with $\mathrm{IC}_{50}$ values estimated to 1.17 and 
$0.75 \mathrm{mM}$ (Table I) respectively. Ferulic acid gave almost the same $\mathrm{IC}_{50}$ value with that reported $(66.3 \mu \mathrm{M})$ by Zhang et al [19] against ADP induction. Gallic and ellagic acid are also widely studied and reported to present several biological activities, such as antibacterial, anti-inflammatory and anticancer [19]-[22]. Ellagic acid exhibits antioxidant, antidiabetic, anticancer and apoptosis-inducing activities [23]. Here, gallic and ellagic acid presented higher inhibition of platelet activation, than ferulic acid, with $\mathrm{IC}_{50}$ values at 0.138 and $0.38 \mathrm{mM}$ respectively (Table I). Fig. 2 shows the effect of gallic acid on (a) ADP- and (b) collagen-activated platelets by Flow Cytometry. The percentage of activated platelets compared to control in platelets incubated with gallic acid at three different concentrations with the expression of CD-61P and CD62-P on a two-dimension dotplot. Similar diagrams were obtained for all the other compounds examined (data not shown).

TABLE I: IC $_{50}$ VALUES (CONCENTRATION OF COMPOUND TESTED IN mM) OF PHENOLIC COMPOUNDS INHIBITION AGAINST ADP AND COLLAGEN

\begin{tabular}{ccc}
\multicolumn{3}{c}{ ACTIVATED PLATELETS } \\
\hline Compound & $\mathrm{IC}_{50}(\mathrm{mM}) \mathrm{ADP}$ & $\mathrm{IC}_{50}(\mathrm{mM})$ Collagen \\
\hline gallic acid & 0.14 & 0.54 \\
ellagic acid & 0.38 & 0.4 \\
ferulic acid & 1.17 & 1.1 \\
aspirin & 0.1 & 0.21 \\
quercetin & 2.2 & 2.26 \\
kaempherol & 0.11 & 0.13 \\
\hline
\end{tabular}
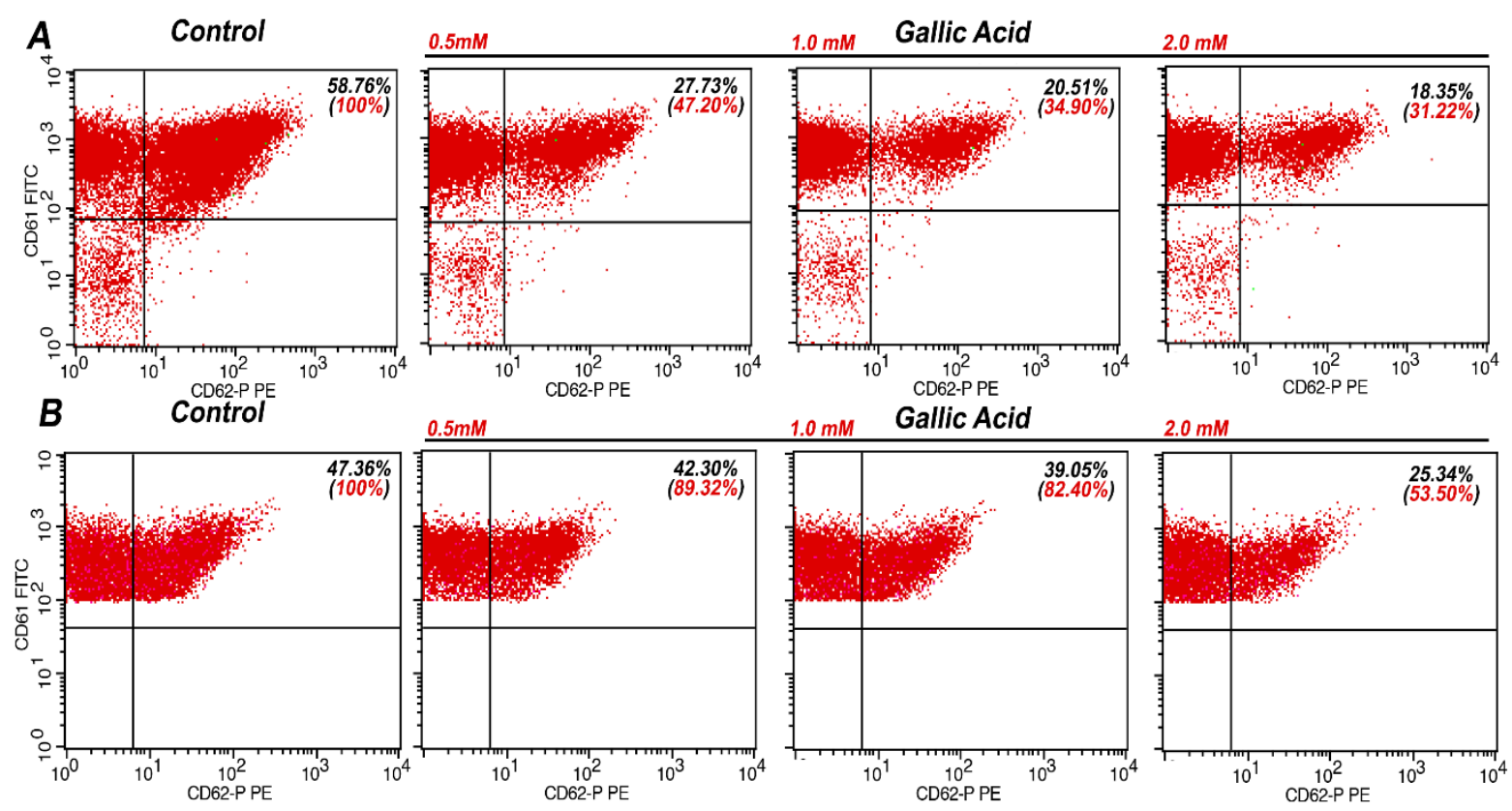

Fig. 2. Calculation of the effect of gallic acid on (a) ADP- and (b) collagen-activated platelets by Flow Cytometry. The percentage of activated platelets compared to control in platelets incubated with gallic acid at indicated concentrations. The expression of CD-61P and CD62-P are shown on a twodimension dot-plot. The results of a representative flow cytometry experiment are presented.

From the two flavonols tested here, kaempferol found to exert stronger antiplatelet activity of ADP stimulated aggregation compared to that of quercetin with $\mathrm{IC}_{50}$ values at 0.11 and $2.2 \mathrm{mM}$ respectively (Table I). Fig. 3 shows a representative $\mathrm{IC}_{50}$ calculation, based on inhibition data from kaempferol on ADP-activated platelets. Interestingly, kaempferol at higher concentrations of $1 \mathrm{mM}$ showed a reversible effect.

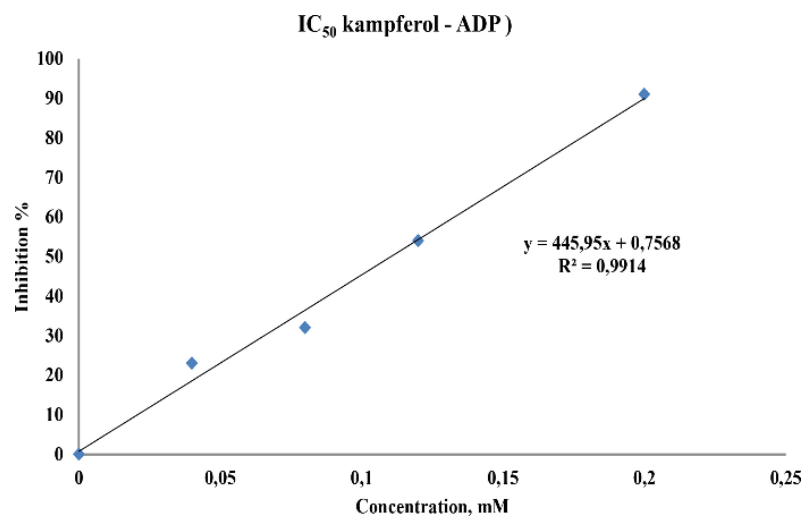

Fig. 3. Percentage platelet inhibition in the presence of different concentrations of kaempferol, in ADP-activated platelets.
As it has been reported by Guo et al [24], quercetin inhibited ADP-induced rat platelet aggregation by $68.33 \pm 2.43 \%$, at a concentration of $331 \mu \mathrm{M}$, while Bijak et al [25] reported a dose-depended decrease of the thrombininduced platelet aggregation by quercetin. Our results showed also a dose-dependent inhibition of platelet reactivity by all five compounds examined. Indicatively, a dose dependent effect of antiplatelet activity is shown in Figure 3 by kaempferol. Similar results were obtained when collagen was used as platelet agonist with $\mathrm{IC}_{50}$ values slightly higher compared to those obtained with ADP (Table I). Gallic acid for example gave $\mathrm{IC}_{50}$ at $0.54 \mathrm{mM}$ with collagen, while the correspondent ADP value was $0.14 \mathrm{mM}$. For comparison, aspirin was tested as a reference compound and similar results were obtained ( $\mathrm{IC}_{50}$ of aspirin with collagen as inducer found at $0.21 \mathrm{mM}$ while the correspondent value with ADP as agonist was $0.1 \mathrm{mM}$ ) supporting that the slightly lower $\mathrm{IC}_{50}$ values, which were obtained using ADP as aggregation agent, than collagen, could be explained since ADP is considered a mild platelet agonist. 
The obtained results from flow cytometry measurements enhanced our previous findings on the inhibition of platelet activation by the five phenolic compounds [10] with some small differences in their ranking. The differences were in phenolic acids with gallic acid as the most potent one. As it can be seen from Table I, gallic acid and kaempherol were the most potent ones. Kaempferol presented higher inhibition of platelet activation than quercetin under the results from aggregometry measurements [11] and was the most potent than all phenolic compounds examined, which gives another perspective to the study. Figure 4 shows the histograms recorded for platelets incubated with kaempferol (at the lower and higher concentration tested) with ADPactivated platelets.

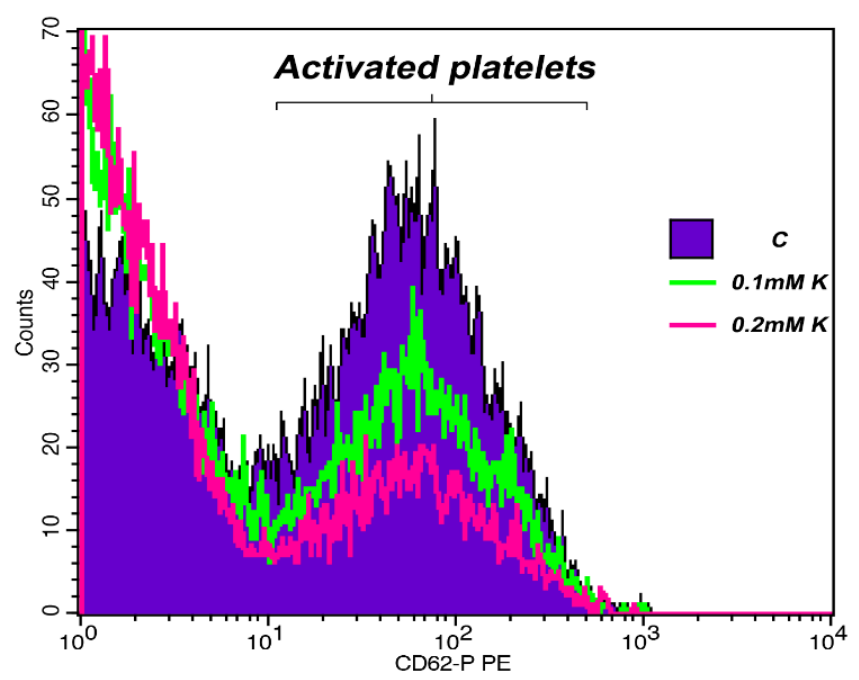

Fig. 4. The effect of kaempferol on ADP-activated platelets by Flow Cytometry. Overlay shows the percentage of ADP-activated platelets

(based on CD-62P expression) in control (Blue Histogram) versus platelets incubated with 0.1 and $0.2 \mathrm{mM}$ kaempferol (Green and pink line,

respectively). The results of a representative flow cytometry experiment are presented.

The antiplatelet activity and cardioprotective effect of quercetin have been also reported [26]-[28]. The interest in the research on flavonoids from plant sources due to their health benefits has been shown in several epidemiological studies. Flavonols, a subcategory of flavonoids, have shown antioxidative activity, anti-inflammatory activity, and antibacterial activity and are commonly found in plants as glycosides. Based on previous reports, a high intake of foods rich in kaempferol reduce the risk of developing several types of cancer including lung, gastric, pancreatic, and ovarian cancer, and of cardiovascular diseases [29]-[32]. The results enhance further the significance of kaempferol for its antiplatelet activity in addition to the other ones previously reported. It is of note that, for our experiments here, whole blood was used aggregated with hirudin and as previously reported blood coagulators affect somehow platelet reactivity and therefore the small differences in $\mathrm{IC}_{50}$ values recorded. Platelet function studies in the presence of different anticoagulants in vitro have shown that it may affect platelet responsiveness [31]. Inhibition of platelet aggregation by aspirin was more pronounced in citrated blood compared to hirudin treated blood, in agreement with the concept of art factually enhanced thromboxane generation in media containing low extracellular calcium levels. In blood anti-coagulated with low molecular mass heparin, platelet aggregation to collagen tended to be enhanced as compared to hirudin-treated blood, whereas platelet responses to ADP at a high concentration were slightly reduced.

On the other hand, the surface expression of P-selectin and activated GPIIb/IIIa are both considered sensitive markers of platelet activation. Platelet surface P-selectin interacts with P-selectin glycoprotein ligand-1 on leukocytes, and thereby plays a critical role in tethering these cells to activated platelets [33]. The resulting monocyte-platelet aggregates were shown to be elevated in myocardial infarction and stable coronary artery disease [34], [35]. Activated GPIIb/IIIa mediates the interaction of platelets with coagulation factors and other platelets [36]. Both P-selectin and activated GPIIb/IIIa were recently associated with adverse ischemic outcomes in patients undergoing angioplasty and stenting for the peripheral arterial disease [37]-[39]. Given the major role of platelets in haemostasis and their interaction with cells of the immune system, platelet dysfunction contributes to cardiovascular disease. It is known, patients with intense thrombogenic potential and complex lesions have 5 times more activated platelets (CD63 positive) than normal individuals [3]. ADPinduced platelet reactivity of phenolic compounds by flow cytometry indicated some differences regarding quantitation of the results but as Gremel et al [40] reports, both approaches capture different aspects of platelet function and are therefore not interchangeable in the assessment of agonists'-induced platelet reactivity.

In addition, the study sought to evaluate the results from flow cytometry measurements compared to those obtained from classical aggregation methods. Examining certain isolated phenolic compounds instead of the relative crude plant extracts, has the advantage of gaining a better understanding of their role and design new non-steroid drugs with fewer side effects compared to aspirin, for example, a common and widely used drug for antiplatelet therapy. Aspirin reduces ischaemic stroke and in parallel may cause haemorrhagic stroke and bleeding. Roughly, these methods can be divided into two groups: platelet aggregation tests (i.e., aggregometry), which measure the extent of platelet aggregation in response to AA and ADP [4]-[6], and flow cytometry, which determines the surface expression of platelet activation markers after the addition of agonists (ADP, collagen).

\section{CONCLUSIONS}

Flow cytometry was used to study and compare the effect on platelet reactivity of five phenolic compounds using ADP and collagen as platelets agonists. All of them found to modulate platelet function by decreasing platelet activation and aggregation. Based on the estimated $\mathrm{IC}_{50}$ values of flavonols, kaempferol presented the strongest inhibition followed by quercetin and the three phenolic acids with the order gallic, ellagic, and ferulic acid. The differences, in the $\mathrm{IC}_{50}$ values, recorded between these and reported ones by other classical aggregometry methods could be expected based on the various parameters and conditions used in the specific experimental work. Flow cytometry, as a rather new 
technique, offers several advantages over the other classical methods, by using whole blood and minimizing the time needed for the examination and is suggested for the screening of natural or new synthetic compounds for possible drugs in antiplatelet therapy. Besides, more information on platelet function and physiology of the most active ones could be obtained by measuring more platelet activation biomarkers in addition to P-selectin.

\section{REFERENCES}

[1] L.K. Jennings, "Mechanisms of platelet activation: Need for new strategies to protect against platelet-mediated atherothrombosis,' Thromb Haemostas, vol.103, pp. 248-257, 2009.

[2] B. Jr Nagy, I.B. Debreceni, J. Kappelmayer, "Flow cytometric investigation of classical and alternative platelet activation markers," $J$ of Intern Fed Clin. Chem \& Lab. medic, vol. 23, pp.11124-1134, 2012.

[3] J.K. Cha, M.H. Jeong, J.Y. Jang, H.R. Bae, Y.J. Lim, J.S. Kim, S.H. Kim, and J.W Kim, "Serial measurement of surface expressions of CD63, P-selectin, and CD40 ligand on platelets in atherosclerotic ischemic stroke. A possible role of CD40 ligand on platelets in atherosclerotic ischemic stroke," Cerebrovasc. Dis, vol.16, pp. 376382, 2003.

[4] N.W. Tsai, W.N, Cheng, C.F. Shaw, C.R. Jan, H.W. Chang, C.R. Huang, S.A.D. Chen, Y.C. Chuang, T.H. Lee, and C.H. Lu "Levels and value of platelet activation markers in different subtypes of acute non-cardio-embolic ischemic stroke," Thromb Res, vol. 124, pp. 213 218, 2009.

[5] C.L. Campbelli, S. Smyth, G. Montalescot, and S.R. Steinhubl, "Aspirin dose for the prevention of cardiovascular disease: a systematic review," The J of the Am Med Assoc, vol. 298(18), pp. 2018-2024, 2007.

[6] V.J. Marder, M.H. Rosove, and M. Minning, "Foundation and sites of action of antithrombotic agents, Best Practice and Research," Clin Haematol, vol. 17, pp. 3-22, 2004.

[7] A. Battaglia, E. Stragliotto, F. Heiman, "Indobufen in the prevention of cerebral ischemic attack (TIA): a prospective randomized, controlled study," Thromb and Haemost, vol. 69, pp.1349-1393,1993.

[8] P. Libby, "Mechanisms of acute coronary syndromes and their implications for therapy," The New Eng. J of Medicine, vol. 368 (21) pp. 2004-2013, 2013.

[9] B.E. Nignpense, K.A. Chinkwo, L. Christopher, C.L. Blanchard, and A.B. Santhakumarm, "Polyphenols: Modulators of Platelet Function and Platelet Microparticle Generation?" Int. J. Mol. Sci, vol. 21, pp. 146-153, 2020. https://doi.org/10.3390/ijms21010146

[10] A. Moschona A, K.D. Kyriakidis, A.D. Kleontas, and M. Liakopoulou-Kyriakides, "Comparative study of natural phenolic acids and flavonols as antiplatelet and anti-inflammatory agents," The Grant Medical J, vol. 2, pp. 57-66, 2017.

[11] A. Moschona, K. Rouptsiou, S. Theodoridou, and M. LiakopoulouKyriakides, "Studies on polyphenols from sea buckthorn berries and pomegranate peels extracts. Recovery, bioactivities and encapsulation into polymers," Int. J Pharm \& Biol Sci, vol. 10, pp.158-171, 2020.

[12] D. Menssen, K. Melber, N. Brandt, and E. Thiel, "The Use of Hirudin as Universal Anticoagulant in Haematology. Clinical Chemistry and Blood Grouping," Clin Chem \& Lab Medicine, vol. 39(12), pp. 1267-1277, 2002.

[13] N.H. Wallén, M. Ladjevardi, J. Albert, and A. Bröijersén, "Influence of Different Anticoagulants on Platelet Aggregation in Whole Blood; A Comparison Between Citrate, Low Molecular Mass Heparin and Hirudin," Thromb Res, vol. 87, pp.151-157, 1997.

[14] M.L. Kalb, L. Potura, G. Scharbert, and S.A. Kozek-Langenecker, "The effect of ex vivo anticoagulants on whole blood platelet aggregation," Platelets, vol. 20, pp. 7-11, 2009. doi: 10.1080/09537100802364076 PMID:19172515.

[15] J. Kappelmayer, B. Jr Nagy, K. Miszti-Blasius, Z. Havessy, and H. Sertidi, "The emerging value of P-selectin as a disease marker," Clin Chem Lab Med, vol. 42, pp. 475-486, 2004.

[16] W. Russel, G. Duthle, "Plant secondary metabolites and gut health: the case for phenolic acids," The proceedings of the Nutrition Society, vol. 10(2), pp. 389-396, 2011.

[17] E. Barone, V. Calabrese, and C. Mancuso, "Ferulic acid its therapeutic potential as a hormetin for age-related diseases," Biotechnol, vol. 10, pp. 97-108, 2009.
[18] H.X. Zhang, H. Lin, C. Qu, YP. Tang, N.G. Li, J. Kai, G. Shang, and B. Li, "Design, synthesis, and in vitro antiplatelet aggregation Activities of Ferulic acid derivatives," J of Chem, hindawi publishing corporation, article id 376527, 2015.

[19] M. Modi, T. Goel, T. Das, S. Malik, S. Suri, A.K. Rawat, et al, "Ellagic acid \& gallic acid from Lagerstroemia speciosa L inhibit HIV-1 infection through inhibition of HIV-1 protease \& reverse transcriptase activity," Indian J Med Res, vol. 137, pp. 540-548, 2013.

[20] D. Lee, S. Eom, Y. Kim, H. Kim, M. Yim, S. Lee, et al, "Antibacterial and synergic effects of gallic acid-grafted-chitosan with beta-lactams against methicillin-resistant Staphylococcus aureus (MRSA)," Can J Microbiol, vol. 60, pp. 629-638, 2014.

[21] C. Liao, K. Lai, A. Huang, J. Yang, J. Lin, S. Wu, et al, "Gallic acid inhibits migration and invasion in human osteosarcoma U-2 OS cells through suppressing the matrix metalloproteinase-2/-9, protein kinase $\mathrm{B}$ (PKB) and PKC signaling pathways," Food Chem Toxicol, vol. 50, pp. 1734-1740, 2012.

[22] A. Borges, C. Ferreira, M. Saavedra, and M. Simoes, "Antibacterial activity and mode of action of ferulic and gallic acids against pathogenic bacteria," Microb Drug Resist, vol. 19, pp. 256-265, 2013.

[23] A. Sahebkar, M. Reza Zirak, and A. Sahebkar, "Ellagic Acid: A Logical Lead for Drug Development?" Cur Pharmac Design, vol. 24(2), pp. 106-122, 2018.

[24] C. Guo, S. Liu, Y. Guo, Y. Yin, J. Lin, X. Chen, and MA. Sun, "Comparative function-structural analysis of antiplatet and antiradical activities of flavonoid phytochemicals," J Anim Plant Sci, vol. 4, pp. 926-935, 2014.

[25] M. Bijak, R. Ziewiecki, J. Saluk, M. Ponczek, I. Pawlaczyk, H. Krotkiewski, P. Wachowicz, and P. Nowak, "Thrombin inhibitory activity of some polyphenolic compounds," Med. Chem. Res, vol. 23, pp. 2324-2337, 2014.

[26] G.P. Hubbard, S. Wolffram, J.A. Lovegrove, and J.M. Gibbins, "Ingestion of quercetin inhibits platelet aggregation and essential components of the collagen-stimulated platelet activation pathway in humans," J Thromb Haemost, vol. 2(12), pp. 2138-2145, 2004.

[27] K-H. Lee, E. Park, H-J. Lee, et al, "Effects of daily quercetin-rich supplementation on cardiometabolic risks in male smokers," Nutr Res Pract, vol. 5(01), pp. 28-33, 2011

[28] A.R. Stainer, P. Sasikumar, A.P. Bye, A.J. Unswort, L.M. Holbrook, M. Tindall, J.A. Lovegrove, and J.M. Gibbins, "The Metabolites of the Dietary Flavonoid Quercetin Possess Potent Antithrombotic Activity, and Interact with Aspirin to Enhance Antiplatelet Effects," TH Open, vol. 3(3), pp. e244-e258, 2019.

[29] S.Cid-Ortega, and J.A. Monroy-Rivera, "Extraction of Kaempferol and Its Glycosides Using Supercritical Fluids from Plant Sources: A Review," Food Technol Biotechnol, vol. 56(4), pp. 480-493, 2018.

[30] A.Y. Chen, and Y.C. Chen, "A review of the dietary flavonoid, kaempferol on human health and cancer chemoprevention," Food Chem, vol. 138(4), pp. 2099-2107, 2013 https://doi.org/10.1016/j.foodchem.2012.11.13.

[31] Y. Cui, H. Morgenstern, S. Greenland, D.P. Tashkin, J.T. Mao, L. Cai, et al, "Dietary flavonoid intake and lung cancer -A populationbased case-control study," Cancer, vol. 112 (10), 2241-2248, 2008. https://doi.org/10.1002/cncr.2339831.

[32] J.M. Calderon-Montano, E. Burgos-Morón, C. Pérez-Guerrero, and M. López-Lázaro, "A review on the dietary flavonoid kaempferol," Mini Rev Med Chem, vol. 11(4), pp. 298-344, 2011 https://doi.org/10.2174/138955711795305335.

[33] N.W. Tsai, W.N. Chang, C.F. Shaw, C.R. Jan, C.R. Chang, S.D. Chem, Y.C. Chuang, T.H. Lee, H.C. Wang, and C.H. Lu, "Levels and value of platelet activation markers in different sub types of acutenoncardio-embopolic ischaemic stroke," Thromb Res, vol. 124, pp. 213 218, 2009.

[34] D.J. McCabe, P. Harrison, I.J. Macklie, PS. Sidhu, G. Purdy, A.S Lawrie, H. Watt, M.M. Brown, and S.J. Machin, "Platelet degranulation and monocyte-platelet complex formation are increased in the acute and convalescent phases after ischaemic stroke or transient ischaemic attack," Br J Haematol, vol. 125, pp. 777-787, 2004.

[35] R.A. Preston, J.O. Coffey, B.J. Materson, M. Ledfordand, and A.B. Alonso, "Elevated platelet P-selectin expression and platelet activation in high risk patients with uncontrolled severe hypertension," Atherosclerosis, vol. 192, pp. 148-154, 2007.

[36] M. Lukasik, G. Dworacki, J. Kufel-Grabowska, C. Watala, W Kozubski, "Chronic hyper-reactivity of platelets resulting in enhanced monocyte recruitment in patients after ischaemic stroke," Platelets, vol. 20 , pp. 235-241, 2009. 
[37] S. Fateh-Moghadam, P. Htum, B. Tomandi, D. Sander, K. Stellios, et al, "Hyper responsiveness of platelets in ischaemic stroke," Thromb Haemost, vol. 97, pp. 974-978, 2007.

[38] E. Csongrady, B. Jr Nagy, T. Fulop, Z. Varga, Z. Karanyl, M.T. Magyar, L. Olah, et al, "Increased levels of platelet activation markers are positively associated with carotid wall thickness and other atherosclerotic risk factors in obese patients," Thromb Haemost, vol. 106, pp. 683-692, 2011.

[39] F. Zeigel, S. Stephan, G. Hoheisel, D. Pfeiffer, C. Riehlmann, and M. Koksch, "P-selectin expression, platelet aggregation, and plateletderived microparticle formation are increased in peripheral arterial disease," Blood Coagul Fibrinol, vol. 11, pp.723-728, 2000.

[40] T. Gremmel, R. Koppensteiner, and S. Panze, "Comparison of Aggregometry with Flow Cytometry for the Assessment of AgonistsInduced Platelet Reactivity in Patients on Dual Antiplatelet Therapy," PLoS ONE 10:e0129666, 2015. 